\title{
Descriptive Epidemiology of Pediatric Intracranial Neoplasms in Egypt
}

\author{
Mohamed Ali El-Gaidi \\ Department of Neurosurgery, Kasr Al-Ainy Medical School, Cairo University, Cairo, Egypt
}

\section{Key Words}

Brain tumors · Children · Egypt · Epidemiology •

Intracranial neoplasms

\begin{abstract}
Objective: The characteristics of 451 Egyptian children (aged 0-14 years) with primary intracranial neoplasms were investigated for demographic, clinical, topographical and pathological features using the most recent 2007 Classification of Central Nervous System Tumors. Patients and Methods: This was a retrospective study performed in the Departments of Pediatric Neurosurgery of the Cairo University Hospitals from 2005 to 2008. Results: There was a slight male predominance (51.4\%) observed in our study, and the most affected age group was 5-9 years old (43.2\%). Most of the tumors were confined to a single compartment (infratentorial in $49.7 \%$, supratentorial in $46.6 \%$ ), while $3.8 \%$ of the tumors involved multiple compartments. The most common intracranial tumors were astrocytomas (35\%), medulloblastomas (18.8\%), craniopharyngiomas (11.3\%) and ependymomas (10\%). Pilocytic astrocytomas constituted $55 \%$ of all astrocytomas and $19.3 \%$ of all brain tumors, only slightly ahead of medulloblastomas. Less common types were primitive neuroectodermal tumors ( $2.7 \%)$, followed by meningiomas, germ cell tumors and choroid plexus tumors ( $2.4 \%$ each). According to the International Classification of Diseases for Oncology Coding (ICD-O-4), benign, borderline and malignant
\end{abstract}

tumors constituted 7.54, 36.14 and 56.32\%, respectively. Conclusion: The characteristics of pediatric intracranial tumors in Egypt are generally similar to those reported in the literature, with only minor differences.

Copyright $\odot 2012$ S. Karger AG, Basel

\section{Introduction}

Intracranial neoplasms are the most common solid pediatric neoplasm (second in both number and mortality only to the hematopoietic-lymphoid system neoplasms) and comprise approximately $20 \%$ of all pediatric malignancies [1-4].

Intracranial tumors in childhood differ significantly in their topographical distribution, pathological types, and therapeutic aspects as well as in prognosis and outcome not only from those that present in adolescents and adults, but also throughout different childhood stages. The diverse times of presentation reflect the different dynamics and biological behaviors of individual tumor entities [5].

The incidence of intracranial tumors in children reveals that there are considerable variations among different regions and countries [1-4]. Each year, approximately 30,000-40,000 new cases are diagnosed worldwide [6]. However, little is known about pediatric intracranial tumors in Africa, particularly in Egypt.

\section{KARGER}

Fax +4161306 1234

E-Mail karger@karger.ch

www.karger.com
(C) 2012 S. Karger AG, Basel

$1016-2291 / 11 / 0476-0385 \$ 38.00 / 0$

Accessible online at:

www.karger.com/pne
Dr. Mohamed Ali El-Gaidi, MD

Department of Neurosurgery 26, Faculty of Medicine

Kasr Al-Ainy Medical School, Cairo University

Cairo 11562 (Egypt)

Tel.+202 236672 60, E-Mail mohamedelgaidi@gmail.com 
To the best of the author's knowledge, this is the first descriptive epidemiological survey of pediatric intracranial tumors in Egypt and countries in the Nile River Ba$\sin$. The aim of the current study is to highlight the characteristics of primary childhood intracranial tumors from a leading Egyptian pediatric referral center with regard to age-related gender distribution, clinical presentation, location and pathological types, using the most recent 2007 World Health Organization (WHO) Classification of Tumors of the Central Nervous System (CNS) and the 4 th edition of the International Classification of Diseases for Oncology (ICD-O-4) [7].

\section{Materials and Methods}

Patient records from the Departments of Neurosurgery at Kasr Al-Ainy Medical School between January 2005 and December 2008 were examined retrospectively for cases (maximum age of 14 years) with pathologically proven primary intracranial tumors. Most of the cases ( $\leq 12$ years) were collected from Abo ElReish Children's Hospital, while children between 13 and 14 years were recruited from three other hospitals (Al-Manial University Hospital, Al-Manial Specialized Hospital and New Kasr Al-Ainy Teaching Hospital).

The recommendations of the Consensus Conferences I and II for the definition and registration of brain and CNS tumors in the USA were followed for the inclusion of intracranial tumors in this study $[8,9]$. The included primary intracranial tumors were those originating from the brain tissue, meninges (including the meninges of the skull base, cavernous sinus and foramen magnum), intradural sections of cranial nerves, optic nerve, pineal gland (these tumors were grouped with the supratentorial (ST) compartment), pituitary gland, craniopharyngeal duct, malignant tumors of olfactory mucosa (one case of esthesioneuroblastoma) and skull. Dermoid cysts (8 cases) and epidermoid cysts (3 cases) were also included.

Intracranial arachnoid cysts (61 cases), metastases (5 cases: Ewing sarcoma, hepatoblastoma, neuroblastoma, rhabdomyosarcoma and Wilms tumor), vascular malformations (2 cases: cavernous angioma and vein of Galen aneurysm), retinoblastomas and other orbital tumors (other than optic nerve tumors), scalp tumors (10 cases) and intraspinal tumors (31 cases) were excluded.

Data on 451 patients with primary intracranial tumors were collected, 4 of whom had multiple solitary tumors above and below the tentorium. Two patients had a fourth ventricular medulloblastoma that had metastasized to the ST region after surgery. The other 2 patients had neurofibromatosis (NF): one had multiple meningiomas (NF I), and the other suffered from left cerebellopontine angle schwannoma and multiple meningiomas (NF II). Each of the 4 cases with multiple tumors was counted only once and not duplicated. The rationale for this approach will be addressed in the Discussion.

Each case was analyzed according to sex, age, clinical presentation, location, and pathology. All cases were (re)-classified according to the most recent $2007 \mathrm{WHO}$ and ICD-O-4 Classifications of CNS tumors. The patients were grouped according to the
WHO standard age-at-diagnosis groups (0-4, 5-9 and 10-14 years), and the 0 - to 4 -year age group was further divided into two subgroups (0-24 months and 2-4 years) to capture any differences in their epidemiological aspects. The characteristics of 21 intracranial neoplasms that occurred during the 1st year of life have been recently published [10]. The details of 6 cases -3 ST and 3 infratentorial (IT) - with regard to the clinical presentation and exact anatomical site were missing; however, their demographic and pathological data were available.

\section{Results}

\section{Patient Demographics}

There was a slight overall male predominance with a male-to-female ratio (M/F) of 232/219 (1.06), and the gender distribution varied according to the age group. The gender distribution was equal in the first 2 years of life, whereas a slight male predominance was present in the 2 - to 9-year age groups $(\mathrm{M} / \mathrm{F}=1.13)$. However, a slight female predominance was noted in the 10- to 14-year age group $(M / F=0.97)$ (fig. 1).

The number of patients increased steadily with age: $10.2 \%$ in the first 24 months, $18.9 \%$ between 2 and 4 years, reaching a peak between 5 and 9 years (43\%) and then declining again between 10 and 14 years (27.9\%).

The mean age of all patients at diagnosis was 7 years $( \pm 3.84 \mathrm{SD})[7.2( \pm 3.65 \mathrm{SD})$ for IT tumors, 6.7 ( $\pm 4 \mathrm{SD})$ for ST tumors and $6.2( \pm 3.8 \mathrm{SD})$ for multiple compartment (MC) tumors]. The age distribution, gender ratio and location of different pathological types of tumors are listed in table 1.

\section{Clinical Presentation}

The most frequent manifestations were intracranial hypertension (67\%), eye symptoms (28.3\%), ataxia (19.1\%) and weakness (18.9\%) (table 2). Intracranial hypertension and eye symptoms were the leading symptoms of intracranial tumors in children regardless of tumor location. Approximately one third of patients with IT and MC tumors displayed ataxia; however, ataxia was remarkably rare in patients with ST tumors, in whom weakness and convulsions were more common.

\section{Location}

Two thirds of tumors were located at or extended to the midline structures where the fourth ventricle (30\%) and the suprasellar/diencephalic areas (19\%) represented the most commonly involved sites within the IT and ST regions, respectively (table 3). 
Approximately half of the tumors (49.7\%) were located in the IT region, while $46.5 \%$ were in the ST region; $3.8 \%$ (17 cases) involved more than one compartment. The most common tumor types in the ST/IT regions were craniopharyngiomas $(\mathrm{n}=49)$ and medulloblastomas $(\mathrm{n}=$ 83). The tumors involving MCs were subdivided into two groups; the first were those extending from one compartment to another (13 cases; ST, IT and spinal compartment), and the second group were those involving multiple isolated tumors in two or more compartments (4 cases; 2 metastatic medulloblastomas and $2 \mathrm{NF}$ ).

The distribution of tumors varied according to age groups. There was a higher predilection for the ST location in the 0 - to 4 -year age group that was notable in the first 2 years of life $(\mathrm{ST} / \mathrm{IT}=1.8)$ than in the 2- to 4 -year age group $(\mathrm{ST} / \mathrm{IT}=1.21)$. Afterward, there was an IT predominance that was more marked in the 5- to 9-year age group $(\mathrm{ST} / \mathrm{IT}=0.76)$ than in the 10 - to 14 -year age group $(\mathrm{ST} / \mathrm{IT}=0.86)$ (fig. 2).

There was a slight overall male predominance of IT tumors $(\mathrm{M} / \mathrm{F}=1.2)$ noted in all age groups. On the other hand, the gender distribution of the ST tumors was nearly equal $(\mathrm{M} / \mathrm{F}=0.98)$, while there was a female predominance of $\mathrm{MC}$ tumors $(\mathrm{M} / \mathrm{F}=0.7)$.

\section{Pathology}

The most common tumors were astrocytomas (all subtypes; $35 \%$ ), followed by medulloblastomas (18.8\%), then craniopharyngiomas (11.3\%) and ependymomas $(10 \%)$. Less common types were primitive neuroectodermal tumors (PNETs) (2.7\%), followed by meningiomas, germ cell tumors (GCTs) and choroid plexus tumors (2.4\% each), then oligodendrogliomas (2.2\%), dermoid cysts $(1.8 \%)$, neuronal/mixed neuroglial tumors and oligoastrocytomas (1.3\% each) (table 4$)$.

Low-grade tumors (WHO I/II) constituted 62.5\% (41.2 and $21.3 \%$, respectively) of all cases, while the rest were high-grade tumors (WHO III/IV) (37.5\%). According to ICD-O-4 coding, benign (0), borderline (1) and malignant tumors (3) constituted 7.54, 36.14 and 56.32\%, respectively.

Astrocytomas (all subtypes) constituted more than one third of childhood brain tumors and were the most common tumors in all age groups. They were the most common ST tumor, surpassing craniopharyngiomas and were as frequent as medulloblastomas within the IT region. There was an almost equal gender distribution but a slight IT predominance.

Pilocytic astrocytomas ranked first, only slightly ahead of medulloblastomas (19.3 vs. $18.8 \%$, respectively)

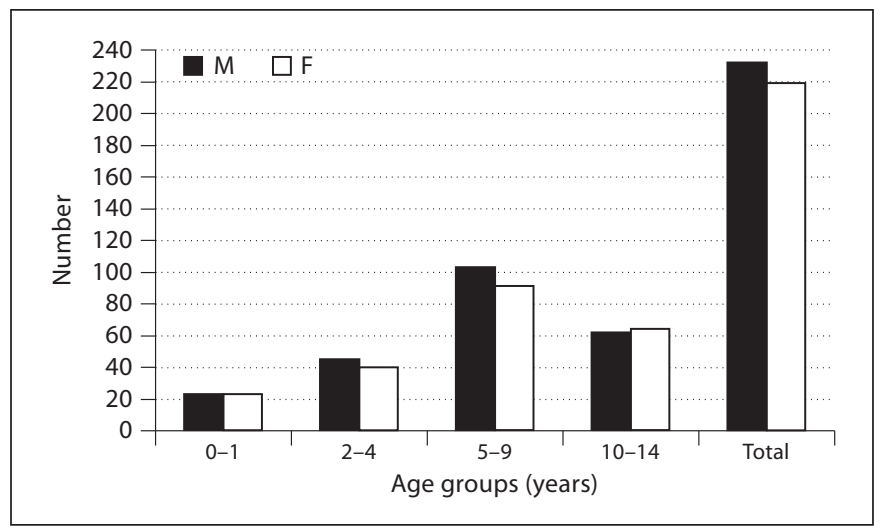

Fig. 1. The gender distribution of 451 intracranial tumors according to age group. $\mathrm{M}=$ Male; $\mathrm{F}=$ female.

and constituted $55 \%$ of all astrocytomas. The gender distribution was virtually equal (M/F $43 / 44=0.96)$; however, there was a slight IT predominance (57.5\%).

Low-grade astrocytomas (WHO I/II), other than pilocytic astrocytomas and high-grade astrocytomas (WHO III/IV), constituted 9.1 and $6.7 \%$, respectively, of all cases. There was no specific gender or location preference in low-grade astrocy tomas; however, there was a slight predominance of high-grade astrocytomas in males and within the ST region.

Embryonal tumors constituted slightly less than one quarter $(23.1 \%)$ of brain tumors in childhood and showed a prominent predilection for the IT region $(87.5 \%$ of cas$\mathrm{es})$, and a slight male predominance $(\mathrm{M} / \mathrm{F}=1.21)$ was noted.

The most common embryonal tumors encountered in this series were medulloblastomas $(n=85)$, which are the most common histological subtype of all IT tumors $(85 / 227=37.4 \%)$. It is noteworthy that 2 cases of medulloblastomas metastasized into the ST region after surgical excision (1 within 6 months and the second after 14 months).

Craniopharyngiomas, the most frequent histological subtype of ST tumors in this series $(n=51)$, constituted $58 \%$ of all suprasellar/diencephalic tumors. All cases of craniopharyngiomas were located in the ST region (specifically at the suprasellar/diencephalon region) with only 2 tumors extending into the prepontine cistern. Craniopharyngiomas prevailed in patients between 2 and 9 years of age (mean $=7.9$ years) with an almost equal gender distribution $(M / F=25 / 26)$. It is intriguing that no single case of craniopharyngiomas was found within the first 24 months of life, despite its assumed congenital origin. 
Table 1. Histopathological data of 451 pediatric tumors according to the 2007 WHO Classification of Tumors of the Central Nervous System

\begin{tabular}{|c|c|c|c|c|c|}
\hline Histological type & $\begin{array}{l}\text { Total } \\
\text { number }\end{array}$ & $\%$ & $\mathrm{M} / \mathrm{F}$ & $\begin{array}{l}\text { Mean age (range) } \\
\text { years }\end{array}$ & $\begin{array}{l}\text { Location } \\
\text { (ST/IT/MC) }\end{array}$ \\
\hline I Neuroepithelial & 242 & 53.7 & $119 / 123$ & $7(0.1-14.9)$ & $119 / 114 / 9$ \\
\hline Astrocytic & 158 & 35 & $80 / 78$ & $7.1(0.5-14.9)$ & $73 / 79 / 6$ \\
\hline Pilocytic astrocytoma (GI) (1) & 87 & 19.3 & $43 / 44$ & $7.3(0.67-14.9)$ & $36 / 50 / 1$ \\
\hline Other low grade I/II & 41 & 9.1 & $21 / 20$ & $6.5(0.5-14.8)$ & $20 / 18 / 3$ \\
\hline Subependymal giant cell astrocytoma (GI) (1) & 2 & & & & \\
\hline Pilomyxoid astrocytoma (GII) (1) & 3 & & & & \\
\hline Diffuse astrocytoma (GII) (3) & 32 & & & & \\
\hline Pleomorphic xanthoastrocytoma (GII) (3) & 4 & & & & \\
\hline High-grade WHO III/IV & 30 & 6.7 & $16 / 14$ & $7.2(1.1-14.9)$ & $17 / 11 / 2$ \\
\hline Anaplastic astrocytoma (GIII) (3) & 17 & & & & \\
\hline Glioblastoma multiforme (GIV) (3) & 10 & & & & \\
\hline Gliosarcoma (GIV) (3) & 2 & & & & \\
\hline Gliomatosis cerebri (GIV) (3) & 1 & & & & \\
\hline Oligodendroglial & 10 & 2.2 & $5 / 5$ & $9.7(6.1-13.6)$ & $9 / 1 /-$ \\
\hline Oligodendroglioma (GII) (3) & 7 & & & & \\
\hline Anaplastic oligodendroglioma (GIII) (3) & 3 & & & & \\
\hline Oligoastrocytoma & 6 & 1.3 & $4 / 2$ & $9.6(5.4-12.5)$ & $5 / 1 /-$ \\
\hline Oligoastrocytoma (GII) (3) & 4 & & & & \\
\hline Anaplastic oligoastrocytoma (GIII) (3) & 2 & & & & \\
\hline Neuronal and mixed neuroglial & 6 & 1.3 & $2 / 4$ & $8.6(4.5-14)$ & $5 / 1 /-$ \\
\hline Desmoplastic gangliocytoma (GI) (0) & 1 & & & & \\
\hline Ganglioglioma (GI) (1) & 3 & & & & \\
\hline Desmoplastic ganglioglioma (GI) (1) & 2 & & & & \\
\hline Ependymal & 47 & 10.4 & $22 / 25$ & $6.7(0.5-14.7)$ & $15 / 32$ \\
\hline Subependymoma (GI) (1) & 2 & & & & \\
\hline Ependymoma (GII) (3) & 37 & & & & \\
\hline Anaplastic ependymoma (GIII) (3) & 8 & & & & \\
\hline Choroid plexus & 11 & 2.4 & $4 / 7$ & $2.7(0.1-11.4)$ & $10 / 1 /-$ \\
\hline Choroid plexus papilloma (GI) $(0)$ & 5 & & & & \\
\hline Atypical choroid plexus papilloma (GII) (1) & 4 & & & & \\
\hline Choroid plexus carcinoma (GIII) (3) & 2 & & & & \\
\hline Pineal & 4 & 0.9 & $2 / 2$ & $5.9(1.1-11.5)$ & $3 /-/ 1$ \\
\hline Pineocytoma (GI) (1) & 1 & & & & \\
\hline Pineal parenchymal tumor of intermediate differentiation (GII) (3) & 1 & & & & \\
\hline Pineoblastoma (GIV) (3) & 2 & & & & \\
\hline II Embryonal & 104 & 23.1 & $57 / 47$ & $6.9(0.75-14.8)$ & $13 / 88 / 3$ \\
\hline Medulloblastoma (GIV) (3) & 85 & 18.8 & $47 / 38$ & $6.6(0.75-14.8)$ & $-/ 83 / 2^{*}$ \\
\hline CNS PNET (GIV) (3) & 15 & 3.3 & & & \\
\hline Not otherwise specified & 12 & 2.7 & & & $9 / 2 / 1$ \\
\hline Medulloepithelioma (GIV) (3) & 2 & & & & \\
\hline Ependymoblastoma (GIV) & 1 & & & & \\
\hline Atypical teratoid/rhabdoid tumor (GIV) (3) & 4 & & & & \\
\hline II Meningeal & 20 & 4.4 & $10 / 10$ & $9.1(0.8-14.5)$ & $11 / 8 / 1$ \\
\hline Nonmeningothelial & 9 & 2 & $6 / 3$ & $6.4(2.5-14.5)$ & $3 / 6$ \\
\hline Mesenchymal & 4 & & & & \\
\hline Hemangiopericytoma II (1) & 1 & & & & \\
\hline Chondrosarcoma (3) & 1 & & & & \\
\hline Ewing sarcoma (3) & 1 & & & & \\
\hline Rhabdomyosarcoma (3) & 1 & & & & \\
\hline Malignant melanoma (3) & 1 & & & & \\
\hline Hemangioblastoma (GI) (1) & 4 & & & & \\
\hline Meningioma (meningothelial) (0) $5,(1) 3$, (3) 3 & 11 & 2.4 & $4 / 7$ & $8.5(0.8-14)$ & $8 / 2 / 1^{*}$ \\
\hline Plus 1 NF II patient with multiple meningiomas and schwannoma & 1 & & & & $(1)^{*}$ \\
\hline
\end{tabular}


Table 1 (continued)

\begin{tabular}{|c|c|c|c|c|c|}
\hline Histological type & $\begin{array}{l}\text { Total } \\
\text { number }\end{array}$ & $\%$ & $\mathrm{M} / \mathrm{F}$ & $\begin{array}{l}\text { Mean age (range) } \\
\text { years }\end{array}$ & $\begin{array}{l}\text { Location } \\
\text { (ST/IT/MC) }\end{array}$ \\
\hline III Cranial nerves & 3 & \multirow[t]{3}{*}{0.7} & \multirow[t]{3}{*}{$1 / 2$} & \multirow[t]{3}{*}{$6.8(5-10.6)$} & \multirow[t]{3}{*}{$-/ 3$} \\
\hline Schwannoma $(\mathrm{GI})(0)$ & 2 & & & & \\
\hline Malignant peripheral nerve sheath tumors (GIII-IV) (3) & 1 & & & & \\
\hline IV Germ cell tumor & 11 & 2.4 & \multirow[t]{7}{*}{$7 / 4$} & \multirow[t]{7}{*}{$6(0-13.3)$} & \multirow[t]{7}{*}{$9 / 1 / 1$} \\
\hline Teratoma & 7 & \multirow[t]{6}{*}{1.6} & & & \\
\hline Mature (0) & 4 & & & & \\
\hline Immature (3) & 2 & & & & \\
\hline Teratoma with malignant transformation (3) & 1 & & & & \\
\hline Germinoma (3) & 3 & & & & \\
\hline Mixed germ cell tumor (3) & 1 & & & & \\
\hline V Sellar & 51 & 11.3 & $25 / 26$ & $7.9(2-14.8)$ & $49 /-/ 2$ \\
\hline \multicolumn{6}{|l|}{ Craniopharyngioma (GI) (1) } \\
\hline VI Lymphomas and hematopoietic neoplasms & 1 & 0.2 & $1 / 0$ & 13 & $1 /-$ \\
\hline Primary lymphoma (3) & & & & & \\
\hline Others (not mentioned in the CNS classification) & 18 & 4 & $11 / 7$ & $7(3.5-14.8)$ & $8 / 10 /-$ \\
\hline Pituitary adenoma $(0)$ & 1 & & & & $1 /-$ \\
\hline Esthesioneuroblastoma (3) & 1 & & & & $1 /-$ \\
\hline Chordoma (3) & 1 & & & & $-/ 1$ \\
\hline Dermoid $(0)$ & 8 & & & & $2 / 6$ \\
\hline Epidermoid (0) & 3 & & & & $-/ 3$ \\
\hline Histiocytosis $(0)$ & 4 & & & & $4 /-$ \\
\hline
\end{tabular}

The histological type of each tumor is followed by the World Health Organization histological grading (I-IV) and the International Coding of Diseases for Oncology $(0,1,3)$ given in italics.

* Cases with multiple solitary tumors.

Ependymomas $(\mathrm{n}=45)$ ranked as the fourth most common brain tumors. Most of the cases (82.2\%) were grade II, and 8 of the cases were anaplastic ependymomas III. There was a marked predilection for the IT region (71\%) and a slight predominance of the female gender (53.3\%).

PNETs, not otherwise specified, were the fifth most common brain tumors and the second most common embryonal tumors in this series (2.7\%). They had a marked predilection for the ST location (75\%), with most cases encountered in the cerebral hemisphere (5/12) and the intraventricular area (2/12). Three cases were found in the IT region, all of which were extra-axial, 2 in the cerebellopontine angle and 1 retroclival, which extended to the second cervical vertebra. There was an equal gender distribution, and one quarter of the cases had occurred during the first 2 years of life.

Meningiomas $(\mathrm{n}=11)$ represented $2.4 \%$ of total cases (apart from an NF II patient with multiple meningiomas associated with a cerebellopontine angle schwannoma). According to the ICD-O- 4 classification, 5 cases were be- nign, 3 were borderline and 3 were malignant. Nine cases were extra-axial, while 2 were present in the lateral ventricle. There was a marked predilection for the ST compartment $(\mathrm{ST} / \mathrm{IT}=8 / 2)$ but a slight female predominance (63.6\%). All cases had a solitary meningioma except for 1 patient (NF I) who presented with multiple meningiomas in the ST/IT regions.

GCTs $(n=11 ; 7$ cases of teratomas of variable differentiation, 3 germinomas and 1 case of a mixed GCT) represented $2.44 \%$ of total cases. Most of the cases $(n=9)$ were ST, and the pineal region was the most frequent site. There was a slight male predominance $(\mathrm{M} / \mathrm{F}=1.75)$.

Choroid plexus tumors ( $\mathrm{n}=11 ; 5$ papillomas I, 4 atypical papillomas II and 2 carcinomas III) had a marked predilection for the ST ventricular system $(\mathrm{ST} / \mathrm{IT}=10 / 1)$ and a moderate female predominance (63.6\%). They were more commonly found in the first 2 years of life $(7 / 11=63.6 \%)$ where they were the most common ST tumors at this age, occurring with the same frequency as medulloblastomas and pilocytic astrocytomas. 
Table 2. Distribution of major symptoms according to tumor location

\begin{tabular}{|c|c|c|c|c|}
\hline Symptoms & $\begin{array}{l}\text { ST } \\
(\mathrm{n}=207)\end{array}$ & $\begin{array}{l}\text { IT } \\
(\mathrm{n}=221)\end{array}$ & $\begin{array}{l}\text { MC } \\
(\mathrm{n}=17)\end{array}$ & $\begin{array}{l}\text { Total } \\
(\mathrm{n}=445)\end{array}$ \\
\hline Intracranial hypertension & $102(49.3 \%)$ & $184(83.3 \%)$ & $12(70.6 \%)$ & $298(67 \%)$ \\
\hline Headache & $95(45.9 \%)$ & $167(75.6 \%)$ & 6 & $268(60.2 \%)$ \\
\hline Vomiting & $27(13 \%)$ & $171(77.4 \%)$ & 6 & $204(45.8 \%)$ \\
\hline Macrocephaly & 11 & 4 & 1 & 16 \\
\hline Disturbed conscious level & 3 & 6 & - & 9 \\
\hline Eye symptoms & $64(30.9 \%)$ & $55(24.9 \%)$ & $7(41.2 \%)$ & $126(28.3 \%)$ \\
\hline Diminution of vision & 60 & 24 & 4 & $88(19.8 \%)$ \\
\hline Squint & 9 & 31 & 2 & $42(9.4 \%)$ \\
\hline Ptosis & 3 & 8 & 2 & 13 \\
\hline Opthalmoplegia & 3 & 2 & - & 5 \\
\hline Parinaud syndrome & 2 & 2 & 1 & 5 \\
\hline Exophthalmos & 3 & - & & 3 \\
\hline Ataxia & $5(2.4 \%)$ & $74(33 \%)$ & $6(35.3 \%)$ & $85(19.1 \%)$ \\
\hline Weakness & $55(26.6 \%)$ & $23(10.4 \%)$ & $6(35.3 \%)$ & $84(18.9 \%)$ \\
\hline Convulsions & $24(11.6 \%)$ & $3(1.4 \%)$ & $2(11.8 \%)$ & $29(6.5 \%)$ \\
\hline Other cranial nerve affection & - & $24(10.9 \%)$ & $3(17.6 \%)$ & $27(6.1 \%)$ \\
\hline Bulbar & & 17 & 2 & \\
\hline Facial & & 7 & 1 & \\
\hline Head tilt & & 2 & & \\
\hline \multicolumn{5}{|l|}{ Miscellaneous } \\
\hline (dysphasia, incontinence, polyuria, growth disturbance) & $11(5.3 \%)$ & $8(3.6 \%)$ & $1(5.9 \%)$ & $20(4.5 \%)$ \\
\hline
\end{tabular}

The total number of presenting symptoms exceeds the total number of patients and the number within each category because many patients presented with more than one symptom. The symptoms of 6 cases were not available (3 ST and 3 IT cases). ST = Supratentorial; IT = infratentorial; $\mathrm{MC}=$ multiple compartments.

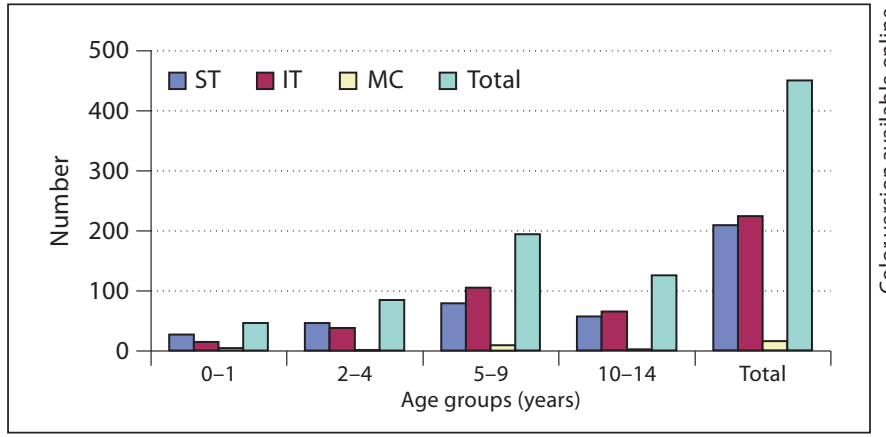

Fig. 2. The distribution of intracranial tumors according to age group and location. ST = Supratentorial; IT = infratentorial; $\mathrm{MC}=$ multiple compartments.

\section{Discussion}

The incidence of CNS tumors in children reveals considerable variations among different regions and countries (11.2 per million per year in India, 12 in Zimbabwe,
16.8 in Turkey, 17.4 in Costa Rica, 25 in Japan, 29.9 in Europe, 32.5 in the USA and 42.8 in Sweden $[1-4,11])$. According to data from the Middle East Cancer Consortium, the age-standardized incidence rate of CNS malignant tumors in Egyptian children (age less than 15 years) was 16.9 per million per year, which was second to lymphoma (37.7) and leukemia (31.9) during the period from 1999 to 2001 [12].

It is evident that the incidence in developing countries is much lower, usually below 20 per million, than in developed countries. The lower incidence of pediatric intracranial tumors in developing countries may be related to a decreased exposure to potential risk factors or may simply be an underestimation due to underreporting or the restricted availability of modern diagnostic techniques [13].

\section{Demographic Data}

Consistent with the vast majority of international studies [11, 14-30], a slight male predominance of childhood intracranial tumors $(\mathrm{M} / \mathrm{F}=1.04)$ was noted in our Egyptian referral center. In this context, Rickert and Pau- 
Table 3. Distribution of tumors according to exact neuroanatomical site

\begin{tabular}{|c|c|c|}
\hline Site & $\mathrm{n}$ & Pathology \\
\hline ST & 210 & \\
\hline SS/chiasmal/hypothalamic & 84 & cran 46 , piloastr 30 , astr I/II 2, dermoid 2, other 4 (PNET, oligastr, astr III/IV, teratoma) \\
\hline Sellar/SS & 2 & cran 2 \\
\hline Sellar & 1 & pituitary adenoma 1 \\
\hline Hemispheric & 42 & $\begin{array}{l}\text { other astr I/II 7, astr III/IV 7, oligdend 7, PNET 5, epend 4, oligastr 3, ganglioglioma 2, } \\
\text { medulloepithelioma 2, teratoma } 2 \text {, other } 3 \text { (desmoplastic gangliocytoma, lymphoma, piloastr) }\end{array}$ \\
\hline Intraventricular & 34 & \\
\hline Lateral & 23 & epend 7, subepend 2, CPT 7, astr I/II 3, men 2, astr III/IV 1, teratoma 1 \\
\hline $3 \mathrm{rd}$ & 6 & CPT 3, other 3 (astr I/II, cran, astr III/IV) \\
\hline Both & 5 & PNET 2, other 3 (teratoma, gangliogioma, ATRT) \\
\hline Thalamus/basal ganglia & 18 & $\begin{array}{l}\text { other astr I/II 7, astr III/IV 6, oligdend 2, other } 3 \text { (malignant melanoma, ependymoblastoma, } \\
\text { oligastr) }\end{array}$ \\
\hline Pineal & 9 & germinomas 3 , pineocytes 3 , teratoma 1 , epend 1 , astr III/IV 1 \\
\hline Orbit & 5 & piloastr 4 (one of them had NF I), men 1 \\
\hline Dural/extradural & 12 & men 5 , histocytosis 4 , mesenchymal 2 , esthesioneuroblastoma 1 (+2 metastatic MB) \\
\hline ST not specified & 3 & PNET 1, piloastr 1, ganglioglioma 1 \\
\hline IT & 224 & \\
\hline 4th ventricle & 133 & $\begin{array}{l}\text { MB 74, epend 29, piloastr 12, other astr I/II 4, astr III/IV 2, dermoid 6, ATRT 2, other } 4 \\
\text { (CPP, ganglioglioma, mixed GCT, epidermoid) }\end{array}$ \\
\hline Cerebellum & 44 & \\
\hline Hemisphere & 34 & $\begin{array}{l}\text { piloastr } 18 \text {, other astr I/II 5, astr III/IV 4, MB 3, hemangioblastoma 2, oligodendroglioma 1, } \\
\text { ATRT } 1\end{array}$ \\
\hline Vermis & 10 & piloastr 9, other astr I/II 1 \\
\hline Cerebellar and 4th V & 13 & piloastr 4, other astr I/II 4, MB 4, epend 1 \\
\hline Brain stem & 19 & piloastr 6 , astr III/IV 5, other astr I/II 4, hemangioblastoma 2, oligoastr 1, epend 1 \\
\hline Extra-axial & 12 & \\
\hline CPA & 7 & schwannoma 3, epidermoid 2, PNET 2 \\
\hline Other sites & 5 & men 2 , mesenchymal 2 , chordoma 1 \\
\hline IT not specified & 3 & MB 2, piloastr 1 \\
\hline MC & 17 & \\
\hline $\begin{array}{l}\text { Extension from one } \\
\text { compartment to another }\end{array}$ & 13 & \\
\hline ST extend IT & 6 & $\begin{array}{l}\text { cran } 2 \text { (SS to prepontine), pineal body } 1 \text { (pineal extend IT), teratoma } 1 \text { (hemispheric } \\
\text { straddled IT), epend } 1 \text { (hemispheric extend to brain stem), astr II (thalamic/basal gang/lat V } \\
\text { to 3rd V, mid-brain) }\end{array}$ \\
\hline IT extend ST & 3 & astrocytoma 3 (grade III 2 , grade II 1 ) (brain stem/4th V to pineal) \\
\hline IT extend SC & 4 & $\begin{array}{l}\text { piloastr } 1 \text {, astr II } 1 \text {, epend III } 1 \text { ( brain stem extending inferior to cervicomedullary junction), } \\
\text { PNET (retroclival to C2) }\end{array}$ \\
\hline Multiple isolated lesions & 4 & $\begin{array}{l}\text { synchronous 2: NF I 1(multiple meningiomas), NF II 1(schwannoma and multiple } \\
\text { meningiomas) metasynchronous 2: MB 2(4th V) metastasized ST }\end{array}$ \\
\hline
\end{tabular}

astr = Astrocytoma; ATRT = atypical teratoid rhabdoid tumor; CPA = cerebellopontine angle; CPT = choroid plexus tumors; cran = craniopharyngioma; epend = ependymoma; $\mathrm{MB}=$ medulloblastoma; men = meningioma; oligastr = oligoastrocytoma; oligdend = oligodendroglioma; piloastr = pilocytic astrocytoma; SC = spinal cord; SS = suprasellar; subepend = subependymoma; $\mathrm{V}=$ ventricle.

lus [5], in their meta-analysis of 10,582 childhood brain tumors accumulated from 16 international surveys, reported that the M/F ratio was 1.29 . Conversely, a few studies, albeit with smaller numbers of patients, have reported a slight female predominance [31, 32].

Pediatric Intracranial Neoplasms in Egypt

\section{Clinical Presentation}

Intracranial hypertension (67\%) and eye symptoms (28.3\%) were the leading clinical presentations of intracranial tumors in this survey, regardless of their location. Intracranial hypertension was more common in poste- 
Table 4. The 7 most common pediatric intracranial tumors by age groups

\begin{tabular}{|c|c|c|c|c|c|}
\hline$\leq 2$ years $(n=46)$ & $2-4$ years $(n=85)$ & $0-4$ years $(n=131)$ & $5-9$ years $(\mathrm{n}=194)$ & $10-14$ years $(n=126)$ & $0-14$ years $(n=451)$ \\
\hline $\begin{array}{l}\text { Piloastr } 7 \\
(15.2 \%)\end{array}$ & $\begin{array}{l}\text { Piloastr } 16 \\
(18.8 \%)\end{array}$ & $\begin{array}{l}\text { Piloastr } 23 \\
(17.6 \%)\end{array}$ & $\begin{array}{l}\text { MB 36 } \\
(18.6 \%)\end{array}$ & $\begin{array}{l}\text { MB } 31 \\
(24.5 \%)\end{array}$ & $\begin{array}{l}\text { Piloastr } 87 \\
(19.3 \%)\end{array}$ \\
\hline $\begin{array}{l}\text { MB 7 } \\
(15.2 \%)\end{array}$ & $\begin{array}{l}\text { Cran 16 } \\
(18.8 \%)\end{array}$ & $\begin{array}{l}\text { MB 18 } \\
(13.8 \%)\end{array}$ & $\begin{array}{l}\text { Piloastr } 35 \\
(18 \%)\end{array}$ & $\begin{array}{l}\text { Piloastr } 29 \\
(23 \%)\end{array}$ & $\begin{array}{l}\text { MB 85 } \\
(18.8 \%)\end{array}$ \\
\hline $\begin{array}{l}\text { CPT } 7 \\
(15.2 \%)\end{array}$ & $\begin{array}{l}\text { MB 11 } \\
(12.9 \%)\end{array}$ & $\begin{array}{l}\text { Cran } 16 \\
(12.2 \%)\end{array}$ & $\begin{array}{l}\text { Cran } 22 \\
(11.3 \%)\end{array}$ & $\begin{array}{l}\text { Cran } 13 \\
(10.3 \%)\end{array}$ & $\begin{array}{l}\text { Cran } 51 \\
(11.3 \%)\end{array}$ \\
\hline $\begin{array}{l}\text { Epend } 5 \\
(10.9 \%)\end{array}$ & $\begin{array}{l}\text { Epend } 9 \\
(10.6)\end{array}$ & $\begin{array}{l}\text { Epend } 14 \\
(10.7 \%)\end{array}$ & $\begin{array}{l}\text { Epend } 21 \\
(10.8 \%)\end{array}$ & $\begin{array}{l}\text { Epend } 10 \\
(7.9 \%)\end{array}$ & $\begin{array}{l}\text { Epend } 45 \\
(10 \%)\end{array}$ \\
\hline $\begin{array}{l}\text { Teratoma } 5 \\
(10.9 \%)\end{array}$ & $\begin{array}{l}\text { Other low-grade } \\
\text { Astr } 8(9.4 \%)\end{array}$ & $\begin{array}{l}\text { Other low-grade } \\
\text { Astr } 12(9.1 \%)\end{array}$ & $\begin{array}{l}\text { Other low-grade } \\
\text { Astr } 20(10.3 \%)\end{array}$ & $\begin{array}{l}\text { Other low-grade } \\
\text { Astr } 9(7.1 \%)\end{array}$ & $\begin{array}{l}\text { Other low-grade } \\
\text { Astr } 41(9.1 \%)\end{array}$ \\
\hline $\begin{array}{l}\text { Other low-grade } \\
\text { Astr } 4(8.7 \%)\end{array}$ & $\begin{array}{l}\text { High-grade Astr, } \\
\text { teratoma, and PNET } \\
3 \text { each }(3.5 \%)\end{array}$ & $\begin{array}{l}\text { CPT } 9 \\
(6.9 \%)\end{array}$ & $\begin{array}{l}\text { High-grade } \\
\text { Astr } 18(9.3 \%)\end{array}$ & $\begin{array}{l}\text { High-grade } \\
\text { Astr } 6(4.8 \%)\end{array}$ & $\begin{array}{l}\text { High-grade } \\
\text { Astr } 30(6.7 \%)\end{array}$ \\
\hline $\begin{array}{l}\text { High-grade } \\
\text { Astr } 3(6.5 \%)\end{array}$ & & $\begin{array}{l}\text { Teratoma } 8 \\
(6.1 \%)\end{array}$ & $\begin{array}{l}\text { Oligodend } 6 \\
(3.1 \%)\end{array}$ & $\begin{array}{l}\text { Oligdend, Olig- } \\
\text { astr } 4 \text { each }(3.2 \%)\end{array}$ & $\begin{array}{l}\text { PNET } 12 \\
(2.7 \%)\end{array}$ \\
\hline Other 8 & Other 16 & Other 30 & Other 36 & Other 20 & Other 100 \\
\hline
\end{tabular}

Astr = Astrocytoma; $\mathrm{CPT}=$ choroid plexus tumors; Cran = craniopharyngioma; Epend = ependymoma; $\mathrm{MB}=$ medulloblastoma; Men = meningioma; Oligastr $=$ oligoastrocytoma; Oligdend $=$ oligodendroglioma; Piloastr $=$ pilocytic astrocytoma .

rior fossa tumors (83.8\%) than in ST tumors (49.3\%). The prevalence of intracranial hypertension in posterior fossa tumors coincides with the incidence of hydrocephalus (84.3\%), which may be the cause in most cases of this limited size compartment. In ST tumors, hydrocephalus alone does not account for this problem because it was noted in only $29.2 \%$ of patients. Therefore, other factors such as a large tumor mass in this capacious compartment may be responsible in other cases.

Vomiting was the most frequent presentation in posterior fossa tumors, slightly ahead of headache (77.4 vs. $75.6 \%$, respectively). On the other hand, headaches were much more prevalent than vomiting in ST tumor patients (46 vs. $13 \%$, respectively). These findings are highly consistent with the data of a recent meta-analysis of 4,171 children with intracranial tumors [33], where the most common presentation of IT tumors was vomiting (75\%), followed by headache (67\%), while unspecified symptoms and signs of raised intracranial pressure were noted in $47 \%$ of ST tumor patients.

Eye symptoms such as diminution of vision, squint, ptosis and ophthalmoplegia were identified early and independent of any manifestations of intracranial hypertension for tumors located or extending into the op- tic apparatus, i.e. orbit, optic nerve or chiasm (suprasellar area) and/or the brainstem, whereas exophthalmos was noted solely in tumors located or extending to the orbit.

Unilateral or bilateral severe diminution of vision (or even blindness) is more common in ST tumors than in IT tumors ( 29 vs. $10.9 \%$, respectively). This symptom is usually the first presentation of optic apparatus tumors, especially in preschool age children. However, for brain tumors located in other areas, eye symptoms usually occur late, after manifestations of intracranial hypertension.

It is worth mentioning that fundus examination was not performed routinely and reliably in all patients in this retrospective study (especially young children, who are generally uncooperative during ophthalmoscopy and may even require sedation). Thus, to avoid inaccurately lower results, fundus abnormalities such as papillodema and optic atrophy were not mentioned in table 2. Papilledema was reported in $13 \%$ of children suffering from brain tumors in the previously mentioned meta-analysis and in approximately $10 \%$ of children with intracranial tumors who were less than 4 years of age. 


\section{Topographical Distribution}

The topographical distribution of intracranial tumors in this study revealed that most of the tumors were confined to a single compartment, while $3.8 \%$ of tumors involved more than one compartment. In accordance with this result, MC tumors accounted for 3.7\% in the Brain Tumor Registry of Japan [18] and 3\% in the US Childhood Brain Tumor Consortium [30]. Tumors involving more than one compartment were not recognized in most previous studies due to a lack of detailed radiological descriptions in most reported surveys.

MC tumors are either an extension of a tumor from one compartment to another (on both sides of the tentorium or involving the spinal canal) or multiple (solitary) tumors. While the former is simply counted as 1 tumor, the latter group is problematic and controversial. Multiple isolated tumors may be of the same morphology (such as multiple meningiomas or metastasis) or of different morphologies. According to the rules of the International Agency for Research on Cancer published in 2004 [34], tumors of the same morphology are counted collectively as 1 tumor, while tumors of different morphologies are counted separately even if they are located in the same compartment.

These rules for counting multiple tumors were followed in this survey with one exception that concerned multiple heterogeneous tumors in an NF II patient. This case was counted as 1 patient and her 'tumor' listed as 'NF2/Schwannoma + Meningioma' because her disease was classified as NF2, and the tumors that develop from that, although histologically identical to common schwannoma and meningioma, have differing and often aggressive, biological behaviors $[35,36]$. 'Two' tumors in 1 patient need to arise entirely independently of each other in order to be counted as such (this is untrue of the NF population) [Frim, pers. commun.].

Many reports document that intracranial tumors have a changing location predilection throughout different childhood stages. In the most common scenario, including the current study, overt ST predominance is noted in the first 2 years of life while substantial IT predominance is evident in the 5- to 9-year age group. In the 2- to 4-year and 10- to 14-year age groups, there is a fairly equal tumor distribution or a slight predominance of one location over the other $[5,30]$. On the other hand, a different pattern is recognized in East Asian countries, where ST tumors predominate in all age groups in Japan [18], in all age groups except for the first 3 years of life in China [19] and in all age groups except the 5- to 9-year age group in Taiwan [20]. During adolescence (15-19 years), a clear ST predominance is evident, as it is in adulthood $[5,18,30]$.
There is an international controversy concerning the predominant location of pediatric brain tumors. Consistent with the slight IT preponderance seen in the present study $(\mathrm{ST} / \mathrm{IT}=216 / 233=$ ratio of 0.94$)$, many authors have reported a slight predominance of IT region tumors compared to those of the ST compartment (Sweden, Denmark, Kashmir, Pakistan, Syria, South Africa, USA and Barbados) [11, 15, 22-24, 29, 30, 32]. This includes the meta-analysis of 16 international studies where the ST/IT ratio was 0.92 [5]. In other series, there was no specific site of predilection for intracranial tumors (Germany, Tunisia and Mexico) [14, 28, 37]. Conversely, other studies have shown a predominance of the ST location for intracranial tumors (Japan, China, Taiwan, Korea, Iran and Morocco) [18-21, 26, 27].

The high ST predominance in East Asian countries (ST/IT ratio 1.4-1.7) is primarily due to the strikingly high frequency of GCTs in these countries (7.7-14.8\%) [18-21]. Nearly all the GCTs are present above the tentorium, where pineal and/or suprasellar regions harbor about three quarters of the total cases [20,21]. The significant discrepancy in the incidence of GCTs between East Asian countries and other regions may be due to genetic differences [20]. Many structural chromosomal anomalies (both autosomal and sex-linked) have been described in intracranial GCTs. Recently, p14 and c-kit gene mutations have been reported to play a role in the oncogenesis of intracranial germinomas [38].

\section{Pathology}

The most recent WHO classification of CNS tumors is the 4th edition published in 2007, which adopted the fourth version of the ICD-O classification system. The 4th edition of CNS tumors classification is very comprehensive and detailed, describing a few new entities, variants and patterns, such as the atypical choroid plexus papilloma, angiocentric glioma, pituicytoma and spindle cell oncocytoma of the adenohypophysis [7]. However, anterior pituitary (adenohypophysis) tumors, Langerhans cell histiocytosis, esthesioneuroblastomas and chordomas were excluded from this classification and were included under endocrine, lymphoreticular neoplasms, peripheral nervous cell tumors and malignant bone tumors, respectively. Although dermoid/epidermoid cysts were not recognized as neoplasms, dermoid cysts with malignant transformations were included in the 4th edition of the WHO Classification of CNS Tumors.

The ICD-O consists of three axes denoting the topography, morphology, and behavior of tumors. The topography code is a 4 -digit code describing the anatomical 
sites of the neoplasms; another 4-digit code for histological type is added, followed by a 1-digit code for behavior of the neoplasm $(0=$ benign; $1=$ borderline/uncertain, and $3=$ malignant). CNS neoplasm topography codes ranged from C70.0 to C72.9 while the histology codes ranged from 9380 to $9589[7,39,40]$.

Malignant tumors (code 3 in the ICD-O system) constituted $56.3 \%$ of cases in this study. The Surveillance, Epidemiology and End Results Cancer Statistics Review [4] reported that malignant CNS tumors represented $73.5 \%$ of the total incidence of pediatric CNS tumors in the USA between 2004 and 2005, while malignant CNS tumors represented 87\% in European children (age 0-14 years) between 1988 and 1997 [2]. The apparent higher incidence of malignant CNS tumors in the European study is due to the adoption of the 2nd edition of the ICDO classification [39], which assigned a malignant code to pilocytic astrocytomas. In contrast, the Surveillance, Epidemiology and End Results Review and the current study adopted the 3rd and 4th editions of the ICD-O classification [39, 40], both of which assigned a borderline code (code 1) to this common pediatric CNS tumor.

Low-grade tumors (WHO I/II) constituted the majority (63\%) of all cases, and the remainder (37\%) were highgrade tumors (WHO III/IV). These results are consistent with other studies from Tunisia [28], where low-grade tumors constituted $67.3 \%$ of all cases. However, other studies from Germany, Syria and Denmark have reported a slight predominance of low-grade tumors $(51.5,53.5$ and $54.5 \%$, respectively) $[5,24,15]$.

Regarding the most common pediatric intracranial tumor, the vast majority of studies $[5,11,12,14-17,20-22$, $25-30,32,37]$ have reported that astrocytomas followed by medulloblastomas are the 2 most common intracranial tumors during childhood. However, two hospitalbased Pakistani and Syrian studies reported medulloblastomas to be the most common pediatric intracranial tumors ahead of astrocytomas [23, 24].

Conversely, craniopharyngiomas were the second most common pediatric brain tumors, after astrocytomas and surpassing medulloblastomas, in Chinese and Nigerian studies [19, 31]. However, GCTs were more common than medulloblastomas (and less frequent than astrocytomas) in a Japanese survey ( 14.8 vs. 11.3 vs. $26.8 \%$, respectively) [18].

Although CNS lymphomas are rare in most studies (including the current study, at only $0.2 \%$ ), CNS involvement by Burkitt lymphoma constituted as high as $9.3 \%$ of intracranial tumors in a 2000 Nigerian study [31] and $4.2 \%$ in a 2007 Iranian study [26]. This high prevalence of Burkitt lymphoma in tropical Africa has been attributed to the combined Epstein-Barr virus and Plasmodium falciparum malarial infection associated with other putative cofactors such as mosquito-borne arboviruses and plant tumor promoters [41].

In summary, there are considerable variations among different regions and countries regarding the current incidence of CNS tumors in children. Generally, developing countries have a lower incidence of pediatric intracranial tumors, which may be an underestimation due to underreporting or the restricted availability of modern diagnostic techniques rather than a true lower exposure to potential risk factors. Thus, it is important to establish a population-based, nationwide pediatric brain tumor registry and to issue the appropriate legislation concerning obligatory notification of CNS tumors of all grades by health personnel and facilities. This would be the nucleus of a national cancer registry for all age groups, enhancing the accurate determination and monitoring of all epidemiological aspects of various CNS tumors and all body cancers.

Moreover, there are clear geographical and ethnic variations in the incidences of some specific pathological entities such as GCTs, craniopharyngiomas and lymphomas, which may be due to genetic or certain specific environmental risk factors and warrant further analytical epidemiological studies.

\section{Conclusion}

This study confirms that the characteristics of pediatric intracranial tumors in Egypt are generally similar to those reported in the literature, with only minor differences. It is the author's hope that this study sheds light on the epidemiology of pediatric intracranial tumors in Egypt and other regions of the world and encourages further future prospective analytical population-based studies that monitor the incidence trends, risk factors, survival rates and quality of life of patients with childhood intracranial tumors.

\section{Acknowledgements}

The author acknowledges valuable contributions from Dr. Elia Anis Ishak and Dr. Ahmed Mahmoud Abd El-Aziz (Prof. of Pathology at Cairo University) for revising and (re)-classifying the pathological slides, Prof. D.M. Frim for useful comments and Prof. F.H. Gilles and Prof. E. Steliarova-Foucher for providing useful references. 


\section{References}

1 Parkin DM, Kramarova E, Draper GJ, Masuyer E, Michaelis J, Neglia J, Qureshi S, Stiller CA (eds): International Incidence of Childhood Cancer, vol 2: IARC Scientific Publications No 144. International Agency for Research on Cancer. Lyon, IARC, 1998.

-2 Peris-Bonet R, Martínez-García C, Lacour B, Petrovich S, Giner-Ripoll B, Navajas A, Steliarova-Foucher E: Childhood central nervous system tumours incidence and survival in Europe (1978-1997): report from Automated Childhood Cancer Information System Project. Eur J Cancer 2006;42:2064-2080.

3 Curado MP, Edwards B, Shin HR, Storm H, Ferlay J, Heanue M, Boyle P (eds): Cancer Incidence in Five Continents, vol IX: IARC Scientific Publication No 160. Lyon, IARC, 2007.

4 Ries LAG, Melbert D, Krapcho M, Stinchcomb DG, Howlader N, Horner MJ, Mariotto A, Miller BA, Feuer EJ, Altekruse SF, Lewis DR, Clegg L, Eisner MP, Reichman M, Edwards BK (eds): SEER Cancer Statistics Review, 1975-2005. Bethesda, National Cancer Institute, 2008. http://seer.cancer.gov/ csr/1975_2005/.

5 Rickert $\overline{\mathrm{C}} \mathrm{H}$, Paulus W: Epidemiology of central nervous system tumors in childhood and adolescence based on the new WHO classification. Childs Nerv Syst 2001;17:503-511.

$\checkmark 6$ Bleyer WA: Epidemiologic impact of children with brain tumors. Childs Nerv Syst 1999; 15:758-763.

7 Louis DN, Ohgaki H, Wiestler OD, Cavenee WK (eds): WHO Classification of Tumours of the Central Nervous System. Lyon, ARC, 2007.

$\checkmark 8$ McCarthy BJ, Surawicz T, Bruner JM, Kruchko C, Davis F: Consensus Conference on Brain Tumor Definition for registration. November 10, 2000. Neuro Oncol 2002,4: 134-145.

D9 McCarthy BJ, Kruchko C, Central Brain Tumor Registry of the United States: Consensus conference on cancer registration of brain and central nervous system tumors. Neuro Oncol 2005;4:196-201.

$\checkmark 10$ Elgaidi MA, Eissa EM: Infantile intracranial neoplasms: characteristics and surgical outcomes of a contemporary series of 21 cases in an Egyptian referral center. Pediatr Neurosurg 2010;46:272-282.

$\checkmark 11$ Lannering B, Sandström PE, Holm S, Lundgren J, Pfeifer S, Samuelsson U, Strömberg B, Gustafsson G, Swedish Childhood CNS Tumor Working Group (VCTB): Classification, incidence and survival analyses of children with CNS tumours diagnosed in Sweden 1984-2005. Acta Paediatr 2009;98:1620-1627.

12 Çavdar AO, Kutluk T: Childhood cancer; in Freedman LS, Edwards BK, Ries LAG, Young JL (eds): Cancer Incidence in Four Member Countries (Cyprus, Egypt, Israel, and Jordan) of the Middle East Cancer Consortium (MECC) Compared with US SEER. NIH Pub No 06-5873. Bethesda, National Cancer Institute, 2006. http://mecc.cancer.gov.
13 Inskip PD, Tarone RE, Hatch EE, Wilcosky TC, Fine HA, Black PM, Loeffler JS, Shapiro WR, Selker RG, Linet MS: Sociodemographic indicators and risk of brain tumours. Int J Epidemiol 2003;32:225-233.

14 Kaatsch P, Rickert CH, Kühl J, Schüz J, Michaelis J: Population-based epidemiologic data on brain tumors in German children. Cancer 2001;92:3155-3164.

15 Gjerris F, Agerlin N, Borgesen SE, Buhl L, Haase J, Klinken L, Mortensen AC, Olsen JH, Ovesen N, Reske-Nielsen E, Schmidt K: Epidemiology and prognosis in children treated for intracranial tumors in Denmark 19601984. Childs Nerv Syst 1998;14:302-311.

- 16 Tseng JH, Tseng MY: Survival analysis of children with primary malignant brain tumors in England and Wales: a population based study. Pediatr Neurosurg 2006;2:67-73.

17 Kaderali Z, Lamberti-Pasculli M, Rutka JT: The changing epidemiology of paediatric brain tumours: a review from the Hospital for Sick Children. Childs Nerv Syst 2009;25: 787-793.

18 Committee of Brain Tumor Registry of Japan: Report of Brain Tumor Registry of Japan (1969-1996). Neurol Med Chir (Tokyo) 2003;43(suppl i-vii):1-111.

19 Zhou D, Zhang Y, Liu H, Luo S, Luo L, Dai K: Epidemiology of nervous system tumors in children: a survey of 1,485 cases in Beijing Tiantan Hospital from 2001 to 2005. Pediatr Neurosurg 2008;44:97-103.

20 Wong TT, Ho DM, Chang KP, Yen SH, Guo WY, Chang FC, Liang ML, Pan HC, Chung WY: Primary pediatric brain tumors: statistics of Taipei VGH, Taiwan (1975-2004). Cancer 2005;104:2156-2167.

21 Cho KT, Wang KC, Kim SK, Shin SH, Chi JG, Cho BK: Paediatric brain tumours: statistics of SNUH, Korea (1959-2000). Childs Nerv Syst 2002;18:30-37.

22 Nayil K, Makhdoomi R, Ramzan A, Zahoor S, Rasool M, Wani A, Dhar A, Mubeen B Singh S, Bhat R, Kirmani A: Childhood tumors of the brain: demographic pattern over a ten-year period in the Kashmir valley. Pediatr Neurosurg 2011;47:31-37.

23 Nasir S, Jamila B, Khaleeq S: A retrospective study of primary brain tumors in children under 14 years of age at PIMS, Islamabad. Asian Pac J Cancer Prev 2010;11:1225-1227.

24 Kadri H, Mawla A: Incidence of childhood brain tumours in Syria (1993-2002). Pediatr Neurosurg 2005;41:173-177.

-25 Siqueira EB, Rahm B, Kanaan I, Jallu A: Brain tumors in pediatric patients at King Faisal Specialist Hospital and Research Centre. Surg Neurol 1993;39:443-450.

26 Mehrazin M, Yavari P: Morphological pattern and frequency of intracranial tumors in children. Childs Nerv Syst 2007;23:157-162.

27 Karkouri M, Zafad S, Khattab M, Benjaafar N, El Kacemi H, Sefiani S, Kettani F, Dey S, Soliman AS: Epidemiologic profile of pediat- ric brain tumors in Morocco. Childs Nerv Syst 2010;26:1021-1027.

28 Bellil S, Limaiem F, Mahfoudhi H, Bellil K, Chelly I, Mekni A, Jemel H, Khaldi M, Haouet S, Zitouna M, Kchir N: Descriptive epidemiology of childhood central nervous system tumours in Tunisia: experience of a single institution over a 15-year period (1990-2004). Pediatr Neurosurg 2008;44: 382-387.

29 Peacock WJ, Lazareff JA, Levin CV: Childhood brain tumours in Cape Town. S Afr Med J 1987;71:5-8.

30 Childhood Brain Tumor Consortium: A study of childhood brain tumors based on surgical biopsies from ten North American institutions: sample description. J Neurooncol 1988;6:9-23.

>31 Olasode BJ, Shokunbi MT, Aghadiuno PU: Intracranial neoplasms in Ibadan, Nigeria. East Afr Med J 2000;77:4-8.

$>32$ Lashley PM, Clarke H, Archer EY: Primary pediatric brain tumours in Barbados: 10year analysis (1978-1988). J Trop Pediatr 1991;37:64-66.

33 Wilne S, Collier J, Kennedy C, Koller K, Grundy R, Walker D: Presentation of childhood CNS tumours: a systematic review and meta-analysis. Lancet Oncol 2007;8:685-695.

34 International Agency for Research on Cancer: International Rules for Multiple Primary Cancers, ICD-O, 3rd Edition: IARC Scientific Publications internal report No 2004/02. Lyon, IARC, 2004.

35 Perry A, Giannini C, Raghavan R, Scheithauer BW, Banerjee R, Margraf L, Bowers DC, Lytle RA, Newsham IF, Gutmann DH: Aggressive phenotypic and genotypic features in pediatric and NF2-associated meningiomas: a clinicopathologic study of 53 cases. J Neuropathol Exp Neurol 2001;60:994-1003.

36 Hamada Y, Iwaki T, Fukui M, Tateishi J: A comparative study of embedded nerve tissue in six NF2-associated schwannomas and 17 nonassociated NF2 schwannomas. Surg Neurol 1997;48:395-400.

37 De La Torre Mondragon L, Ridaura Sanz C, Reyes Mujica M, Rueda Franco F: Central nervous system tumors in Mexican children. Childs Nerv Syst 1993;9:260-265.

38 Sato K, Takeuchi H, Kubota T: Pathology of intracranial germ cell tumors. Prog Neurol Surg 2009;23:59-75.

39 Percy C, Holden V, Muir C (eds): International Classification of Diseases for Oncology, ed 2. Geneva, World Health Organization, 1990.

40 Fritz A, Percy C, Jack A, Shanmugaratnam K, Sobin L, Parkin DM, Whelan SL (eds): International Classification of Diseases for Oncology, ed 3. Geneva, World Health Organization, 2000.

41 Van den Bosch CA: Is endemic Burkitt's lymphoma an alliance between three infections and a tumour promoter? Lancet Oncol 2004; 5:738-746. 